\title{
El rol del eWOM en la comunicación de RSC en redes sociales*
}

\author{
Claudia Aguirre ${ }^{* *}$ \\ Salvador Ruiz*** \\ Mariola Palazón ${ }^{* * * x}$ \\ Augusto Rodríguez $z^{* * * *}$ \\ Recibido: 2020-06-02 • Enviado a pares: 2020-06-13 \\ Aprobado por pares: 2020-07-05 • Aceptado: 2020-08-22 \\ https://doi.org/10.22395/angr.v19n38a2
}

\begin{abstract}
Resumen
Las empresas tratan de innovar en sus estrategias de comunicación para ofrecer información tanto sobre sus bienes y servicios, como información corporativa relacionada con las actividades de RSC que la empresa lleva a cabo. En la actualidad, la comunicación sobre RSC es muy importante y poco se sabe acerca de la mejor forma de hacerlo. En este sentido, las redes sociales se han convertido en un importante canal de comunicación entre empresas y consumidores. A través de las redes sociales, los individuos comparten contenido con otros usuarios, pero también pueden apoyar a la empresa con la difusión de la información relativa a sus actividades. Esta difusión de información en entornos electrónicos se conoce como eWOM.

El objetivo de este trabajo es analizar el rol del eWOM en la difusión de la información de RSC de la empresa. Para ello, se realiza un estudio basado en la metodología del análisis de contenido que contrasta si aquellas publicaciones de la empresa en la red social Facebook con contenido de RSC generan más interacciones de los consumidores que las publicaciones con información de tipo comercial. Los resultados obtenidos corroboran que la empresa conseguirá que se difunda más su información en términos de eWOM cuando sus publicaciones contienen información de RSC, puesto que dichas publicaciones logran un mayor número de me gustas y comentarios, a la vez que son compartidas en una mayor medida.

Palabras clave: responsabilidad social; medios sociales; medios electrónicos; comunicación; transferencia de información; consumidor.
\end{abstract}

El presente artículo se ha realizado en el marco de la tesis doctoral titulada Antecedentes y consecuencias de compartir información mediante boca-oído electrónico sobre actividades de responsabilidad social corporativa, en la línea de investigación Comportamiento del consumidor en la Facultad de Ciencias de la Empresa de la Universidad de Murcia, España. Agradecemos el apoyo del proyecto ECO2017-83999-R de la Agencia Estatal de Investigación (AEI) y los Fondos Europeos de Desarrollo Regional (Feder).

. $\quad$ Estudiante de doctorado en Administración, Universidad del Valle, Cali, Colombia; y en Ciencias de la Empresa, Universidad de Murcia, Murcia, España. Investigadora del grupo de investigación en marketing, Universidad del Valle, Cali, Colombia; y de comportamiento del consumidor, Universidad de Murcia, Murcia, España. Correo electrónico: cxaguirrem@gmail.com. Orcid: https://orcid. org/0000-0003-0297-8611.

... Doctor en Ciencias Económicas y Empresariales, Universidad de Murcia, Murcia, España. Catedrático de la Facultad de Economía y Empresa, Universidad de Murcia, Murcia, España. Correo electrónico: salvruiz@um.es. Orcid: https://orcid.org/0000- 0001-7487-8308.

.... Doctora en Ciencias de la Empresa, Universidad de Murcia, Murcia, España. Profesora Titular en la Facultad de Economía y Empresa, Universidad de Murcia, Murcia, España. Correo electrónico: mariolap@um.es. Orcid: https://orcid.org/0000-0001-6957-9653.

..... Doctor en Ciencias de la Empresa, Universidad de Murcia, España. Profesor asociado en la Facultad de Administración, Universidad del Valle, Cali, Colombia. Correo electrónico: augusto.rodriguez@correounivalle.edu.co. Orcid: https://orcid.org/0000-0003-2865-1748. 


\title{
The Role of the eWOM in the RSC Communication in Social networks
}

\begin{abstract}
Companies try to innovate in their communication strategies for offering information about their goods and services and other information related with the RSC activities performed by the company. Currently, RSC communication is very important and little is known about the best way to perform it. Thus, social networks have become an important communication channel between companies and consumers. Through social networks, individuals share information with other users but also support the company in the broadcasting of the information related to their activities. This type of broadcasting in virtual contexts is known as eWOM.

The goal of this research is to analyze the role of the eWOM in the broadcasting of the RSC information of the company. For that, it performs a study based in content analysis that compares if the company's publications in the Facebook social network with RSC content generate more interactions by the consumers than the publications with commercial information.

The results corroborate that the company achieves a greater broadcasting of their information in terms of eWOM when their publications have RSC information, given that those publications achieve a greater number of 'likes' and comments, and are shared more widely at the same time.
\end{abstract}

Keywords: social responsibility; social media; virtual media; communication; information transfer; consumer.

\section{O papel do eWOM na comunicação de RSC em redes sociais}

\begin{abstract}
Resumo
As empresas tentam inovar em suas estratégias de comunicação para oferecer informação tanto sobre seus bens e serviços quanto informação corporativa relacionada com as atividades de RSC que a empresa realiza. Na atualidade, a comunicação sobre RSC é muito importante e pouco se sabe sobre a melhor forma de realizá-la. Nesse sentido, as redes sociais têm se tornado um importante canal de comunicação entre empresas e consumidores. Por meio das redes sociais, os indivíduos compartilham conteúdo com outros usuários e podem apoiar a empresa com a difusão da informação relativa a suas atividades. Essa difusão de informação em ambientes eletrônicos é conhecida como eWOM. Assim, o objetivo deste trabalho é analisar o papel do eWOM na difusão da informação de RSC da empresa. Para isso, é realizado um estudo baseado na metodologia da análise de conteúdo que compara se as publicações da empresa na rede social Facebook com conteúdo de RSC geram mais interações dos consumidores do que as publicações com informação de tipo comercial. Os resultados corroboram que a empresa conseguirá que sua informação seja mais divulgada em termos de eWOM quando suas publicações contiverem informação de RSC, visto que essas publicações atingem maior número de likes e comentários, ao mesmo tempo que são compartilhadas em maior medida.
\end{abstract}

Palavras-chave: responsabilidade social; meios sociais; meios eletrônicos; comunicação; transferência de informação; consumidor. 


\section{Introducción}

Las empresas están cada vez más interesadas en que el contenido de sus comunicaciones corporativas sea de interés para los consumidores. Estos esfuerzos están enfocados en influir positivamente en las percepciones, actitudes y comportamiento de los consumidores hacia la empresa (López, 2018; Song yWen, 2019). Las formas publicitarias tradicionales han dejado de ser eficientes y se abre lugar para nuevas formas de generación de contenido corporativo que permita atraer y vincular al consumidor con la empresa (Kam, Robledo-Dioses y Atarama- Rojas, 2019). En este contexto, se presenta el concepto de boca a boca electrónico (eWOM) como la evolución del boca a boca tradicional (WOM). El eWOM alude a cualquier comunicación positiva o negativa hecha por consumidores, actuales o antiguos sobre un producto, servicio o compañía que se pone a disposición de una multitud de individuos e instituciones a través de internet (Hennig-Thurau et al., 2004). Ha sido la aparición de las redes sociales lo que ha originado un cambio radical en el intercambio de información entre individuos. A través de estas redes, los consumidores han abandonado su rol pasivo de receptores de información (Kim, 2019) y han pasado a tener un rol activo, ya que en las redes sociales pueden intercambiar información sobre bienes, servicios y marcas con otros consumidores o incluso con la propia empresa (Erkan y Evans, 2016). Así, las empresas están valorando los comentarios que los consumidores hacen en las diferentes redes sociales, ya que contienen información que les permite tener elementos reales para innovar e incrementar las experiencias positivas del consumidor con la marca (Kam, Robledo-Dioses y Atarama- Rojas, 2019).

En esta línea, el eWOM ha sido estudiado como una herramienta de persuasión que permite influir tanto en la confianza y la actitud de los consumidores hacia la marca como en la intención de comprar los productos de la empresa (Bhandari y Rodgers, 2018). Por ejemplo, la encuesta de confianza en publicidad a nivel mundial de Nielsen (2015) reporta que el 66 \% de los consumidores confían más en las opiniones que otros consumidores publican en línea. En esta misma línea, Podium (2017) indica que el 93 \% de los consumidores buscan las opiniones de otros antes de tomar la decisión de comprar un producto. De esta forma, las empresas están otorgando cada vez más importancia al desarrollo de nuevas formas de comunicación como interfaces, aplicaciones, páginas web y plataformas de redes sociales (Stryja y Satzger, 2018) para vincular a los consumidores y conseguirque sean ellos mismos quienes difundan las comunicaciones corporativas de contenidos tanto comerciales como no comerciales (Hennig-Thurau et al., 2004).

Por otro lado, varios estudios han demostrado que los consumidores no solo demandan información sobre los bienes y servicios de la empresa, sino también 
sobre otros aspectos como, por ejemplo, las actividades de RSC que se llevan a cabo (Kim, 2019). De acuerdo con la Asociación Española de Contabilidad y Administración de Empresas [AECA] (2004), la RSC se define como:

el compromiso voluntario de las empresas con el desarrollo de la sociedad y la preservación del medio ambiente, desde su composición social y un comportamiento responsable hacia las personas y grupos sociales con quienes se interactúa que centra su atención en la satisfacción de las necesidades de los grupos de interés y que va más allá del mero cumplimiento de la normativa legal establecida y de la obtención de resultados exclusivamente económicos a corto plazo. (p. 21)

De manera más reciente, la Comisión Europea (2011) define la RSC como "la responsabilidad de las empresas por su impacto en la sociedad".

La literatura de RSC sugiere que existe una relación positiva con la innovación empresarial, ya que la RSC se ha convertido en una nueva forma de crear valor comercial. Por ejemplo, ante la preocupación mundial por el medio ambiente, las empresas han innovado en sus procesos productivos principalmente para utilizar menos materia prima, reciclar, reducir el gasto energético, manejo de residuos, reducir la huella de carbono, generar menos impacto ambiental, etc. (Matus, 2018; Suárez-Perales, Rivera-Torres y Garces-Ayerbe, 2018).

El estudio de la RSC ha alcanzado gran relevancia en las áreas de marketing y en la comunicación corporativa, principalmente por su impacto en el comportamiento del consumidor (Matus, 2018; Marin y Ruiz de Maya, 2013). Es ampliamente aceptado que los consumidores se identifican de forma más positiva conempresas que realicen actividades de RSC (Cha, Yi y Bagozzi, 2016). Esto sucederásiempre y cuando los consumidores no perciban que la empresa está realizando la RSC más por motivaciones de tipo egoísta y para lograr un objetivo propio (Lim, 2020). Sin embargo, a pesar de los recursos que las empresas invierten en actividades de RSC y su interés en comunicarlas a través de las memorias anuales de RSC, por ejemplo, una gran proporción de consumidores no son conscientes o desconocen el esfuerzo realizado por la empresa (Cha, Yi y Bagozzi, 2016). Por tanto, las empresas deben plantearse cómo aumentar la efectividad cuando comunican las actividades de RSC, y en qué medida los consumidores tendrán la intención de generar eWOM de dichos mensajes para ayudar a la empresa a difundir el mensaje de RSC

En este sentido, este artículo presenta el eWOM como una herramienta para incrementar la difusión de la comunicación corporativa no comercial, como es la información de RSC. El objetivo de este trabajo es analizar si las publicaciones que realiza una empresa en sus redes sociales generan más interacciones entre sus 
seguidores cuando se incluye información de RSC en comparación a cuando solo incluye información de tipo comercial. Para ello, se ha utilizado la metodología de análisis de contenido para analizar las reacciones que tienen los consumidores ante publicaciones con contenido de RSC y las que no contienen dicha información realizadas por las principales empresas de moda en España en la red social Facebook.

Este artículo se divide en cuatro secciones. En la primera se aborda una revisión de la literatura sobre la evolución del WOM al eWOM junto con las motivaciones para generar eWOM de RSC. En la segunda sección se presenta el diseño metodológico llevado a cabo para realizar el análisis de contenido de las publicaciones de Facebook. En la tercera sección se incluyen los hallazgos. Para finalizar, se desarrollan los apartados de discusión y conclusiones.

\section{Del WOM al eWOM}

Internet ha posibilitado un ilimitado acceso a la información. Por este medio, los consumidores pueden fácilmente solicitar y ofrecer información sobre sus experiencias de consumo. Este intercambio de información permite que los consumidores, basándose en experiencias previas de otros consumidores, se hagan una idea más clara sobre las bondades o debilidades de un producto con el objetivo de realizar una compra (Berger, 2014). En este contexto, el WOM y el eWOM han sido estudiados como una herramienta persuasiva de comunicación que permite influir en las actitudes de los consumidores y sus decisiones de compra (Bhandari y Rodgers, 2018).

La literatura sobre eWOM referencia al WOM como su concepto antecesor (Gvili y Levy, 2016). Por ello, resulta de interés aclarar los aspectos que diferencian a los dos procesos de comunicación y que se derivan del cambio en el canal de comunicación (ver tabla 1). En primer lugar, el canal de comunicación deja de ser cara a cara (WOM) y pasa a situarse en el campo de las redes sociales virtuales (eWOM) (Hennig-Thurau et al., 2004). Asimismo, a diferencia del WOM, donde siempre se conoce quien es el emisor y receptor, en el eWOM la fuente del mensaje puede ser conocida o anónima, tanto para el emisor como para el receptor (Chatterjee, 2001). Además, el tipo de mensaje cambia de oral (WOM) a uno escrito en formato de publicación o comentario en línea (eWOM) (Sen y Lerman, 2007), o incluso se adopta el formato video (Joa, Kim y Ha, 2018). El intercambio de información en el eWOM no ocurre necesariamente de forma sincronizada y bidireccional como en el WOM. Por ejemplo, una vez publicado un comentario en las redes sociales, el emisor puede difundir un mensaje y recibir o no una respuesta del receptor o de los múltiples receptores (Hennig-Thurau et al., 2004). El tamaño de la red es otra diferencia importante puesto que internet permite que la información llegue a un elevado número de receptores (Berger, 2014). 
Las comunicaciones tienen un mayor alcance por el canal y la velocidad de difusión en un contexto de eWOM (López y Sicilia, 2013) en comparación con la comunicación tradicional. Por último, dado el posible anonimato del emisor en el eWOM, puede existir un mayor desconocimiento de si hay un interés comercial en la difusión del mensaje (Chatterjee, 2001). El resumen de las principales diferencias entre WOM y eWOM se muestran en la tabla 1.

Tabla 1. Principales diferencias entre WOM y eWOM

\begin{tabular}{lll}
\hline \multicolumn{1}{c}{ Aspecto } & WOM & \multicolumn{1}{c}{ eWOM } \\
\hline Canal de comunicación & Cara a Cara & Electrónico \\
\hline Fuente del mensaje & Conocido & Conocido o desconocido \\
\hline Tipo de mensaje & Oral & Escrito y video \\
\hline Tipo de comunicación & Sincrónico & Asincrónico \\
\hline Modelo de comunicación & Uno a uno & Muchos a muchos \\
\hline Comunicación & Bidireccional & Bidireccional/ unidireccional \\
\hline Tamaño de la red & Pequeña & Ilimitada \\
\hline Alcance & Limitado & El mundo de internet \\
\hline Interés comercial & Conocido & Conocido o desconocido \\
\hline
\end{tabular}

Fuente: elaboración propia a partir de López y Sicilia (2013).

El surgimiento de las redes sociales ha favorecido los estudios sobre eWOM (Hennig-Thurau et al., 2004). De hecho, el concepto ha sido denominado de formas distintas por diversos estudios. Así, se menciona eWOM con términos diferentes como "online WOM" (Duan, Gu y Whinston, 2008) para referirse a compartir información boca-boca en línea, "sWOM" (Trusov, Bucklin y Pauwels, 2009) como el acto de compartir información boca-boca a través de redes sociales y "word of mouse" (Berger, 2014) para hacer referencia a trasferir información a través de equipos informáticos. En estas investigaciones podemos diferenciar dos grandes corrientes. Un primer grupo de estudios se ha centrado en evaluar el impacto del eWOM en variables relacionadas con el comportamiento del consumidor, tales como intención de compra, lealtad, actitud hacia la empresa y sus productos, así como atracción de nuevos clientes (Alhidari, Iyer y Paswan, 2015). Un segundo grupo de trabajos ha explorado las motivaciones que tienen los consumidores para involucrarse en eWOM (Hennig-Thurau et al., 2004).

Para el primer grupo de estudios, el impacto del eWOM en las variables de comportamiento del consumidor es un hecho constatado (Alhidari, Iyer y Paswan, 
2015). Se afirma que los comentarios compartidos a través de eWOM juegan un papel más influyente, más creíble y más fiable para los consumidores que la información generada por la propia empresa (Trusov, Bucklin y Pauwels, 2009). Existen estudios que demuestran que el intercambio de información sobre experiencias previas de consumo, tanto positivas como negativas, ayuda a los consumidores para tomar una decisión de compra que permite reducir el riesgo de hacer una mala compra (Alhidari, Iyer y Paswan, 2015; King et al., 2014). En este sentido, el propósito de estos estudios es demostrar la utilidad que tienen las valoraciones positivas y negativas difundidas sobre productos, marcas y empresas cuando los nuevos consumidores se enfrentan a una decisión de compra (Moon, Costello y Koo, 2017). Un eWOM negativo proporciona señales de riesgo que sirven como advertencia para que los consumidores eviten el uso de un producto (Bhandari y Rodgers, 2018). De hecho, estudios recientes señalan que aquellas publicaciones que contienen emociones negativas se difunden de manera más amplia y rápida que las que contienen palabras que hacen referencia a emociones positivas (Zhu, Kim y Park, 2020). Por otra parte, un eWOM positivo proporciona señales de seguridad y favorece la adquisición de nuevos clientes (Trusov, Bucklin y Pauwels, 2009). En este mismo grupo de estudios, otros autores se centran en explorar la existencia de una relación positiva entre la disposición que tiene un consumidor a compartir una valoración positiva de un bien o servicio y variables como la identificación con la empresa y la lealtad hacia la marca (King et al., 2014).

Las empresas son conscientes de los efectos positivos del eWOM (Bhandari y Rodgers, 2018) y han incorporado en sus estrategias de marketing digital el potencial de las redes sociales como herramienta de comunicación (Bhandari y Rodgers, 2018; Kwon, Ha y Kowal, 2017). Sin embargo, los estudios que apuntan a explorar esta línea de investigación son más recientes (Alhidari et al., 2015). La información disponible en las redes sociales será creíble en la medida en que el individuo perciba que no hay detrás un interés meramente comercial (Cantallops y Salvi, 2014; Appelman y Sundar, 2016). Las empresas están cada vez más interesadas en utilizar las redes sociales como herramienta para la generación de eWOM. Para ello, se diseñan estrategias de marketing digital cuyo objetivo es incrementar las interacciones de la empresa o marca con los consumidores y de los consumidores entre ellos en redes sociales (Oeldorf-Hirsch y Sundar, 2015).

El segundo grupo de estudios aborda los motivos que tienen los consumidores para generar eWOM. Estos trabajos se han centrado en analizar las razones que llevan a los individuos a compartir información. En este sentido, diversos autores han demostrado que los individuos comparten información por motivaciones de carácter egoísta con el propósito de satisfacer sus necesidades personales (Berger, 
2014; Hennig-Thurau et al., 2004). Además, resulta de interés considerar también si la transmisión de información se deriva o no de una experiencia de consumo (Berger, 2014; Chu, Lien y Cao, 2018). Así, las motivaciones que tienen los consumidores para eWOM se pueden clasificar utilizando dos criterios: a) si el acto de compartir está motivado por la obtención de un beneficio para el propio emisor o el beneficio es para los demás; y b) si el acto de compartir se produce tras una experiencia de consumo o no está necesariamente ligado a la misma (Berger, 2014; Hennig-Thurau et al., 2004).

Las motivaciones que buscan el beneficio propio y que son producto de una experiencia de consumo incluyen i) satisfacer la necesidad de sentirse único o diferente (Veloutsou et al., 2015), ii) recibir incentivos económicos o premios por parte de la empresa (Hennig-Thurau et al., 2004; Sundaram, Mitra y Webster, 1998), iii) ofrecer consejo para intentar resolver un problema, iv) persuadir a otros con el objetivo de influir en la toma de una decisión para comprar un producto (HennigThurau et al., 2004) y v) regular las emociones asociadas a una experiencia de consumo (Berger, 2014). Regular las emociones hace referencia al proceso por el que se compensan emociones positivas y negativas al compartir información con otros individuos (Berger, 2014). Compartir información sobre una experiencia de consumo satisfactoria refuerza los sentimientos de alegría, felicidad y excitación derivados de dicha experiencia, mientras que la experiencia negativa aumenta los sentimientos de ansiedad, depresión y venganza (Sundaram, Mitra y Webster, 1998).

En segundo lugar, las motivaciones que están relacionadas con beneficiar a otros y que son producto de una experiencia de consumo se refieren al i) altruismo, es decir, hacer algo por otros sin esperar nada a cambio o prevenir a otros de una experiencia negativa que el emisor ha tenido (Hennig-Thurau et al., 2004) y ii) a ayudar a la empresa, que generalmente refleja un agradecimiento del comprador como resultado de una experiencia que supera las expectativas del consumidor (Sundaram, Mitra y Webster, 1998).

Entre las motivaciones que buscan el beneficio propio, pero que no necesariamente guardan una relación con actividades de consumo, podemos identificar i) la mejora del autoconcepto, es decir, que el emisor busca asociar a su imagen conceptos positivos como la astucia o la inteligencia para mostrarse ante otros como un buen comprador (Berger, 2014; Sundaram, Mitra y Webster, 1998), ii) mostrar una identidad deseada, puesto que el emisor busca que el receptor de la información infiera aspectos de su identidad (Berger, 2014; Chung y Darke, 2006), y iii)la vinculación social, que se refiere a que los individuos están interesados en permanecer en contacto con otros y, en este sentido, Berger (2014) afirma que buscar vínculos es la motivación más común para mantener una relación de comunicación. 
Finalmente, encontramos aquellas motivaciones relacionadas con beneficiar a otros y que no son resultado de una actividad de consumo (Berger, 2014; HennigThurau et al., 2004). En esta categoría encontramos nuevamente la motivación de ayudar a la empresa, pero ya no se refiere al agradecimiento por la satisfacción con sus productos, sino al agradecimiento del consumidor por alguna actividad realizada por la empresa o un mensaje emitido, por ejemplo, un mensaje de responsabilidad social que el consumidor percibe como positivo y decide ayudar a difundirlo. Este planteamiento se presenta en la tabla 2.

Tabla 2. Motivaciones para eWOM en función de los beneficios buscados y el origen de la experiencia

\begin{tabular}{cll}
\hline Tipo de motivación & \multicolumn{1}{c}{$\begin{array}{c}\text { Derivadas de una experiencia } \\
\text { de consumo }\end{array}$} & $\begin{array}{c}\text { No derivadas de una } \\
\text { experiencia de consumo }\end{array}$ \\
\hline Beneficios para el emisor & $\begin{array}{l}\text { Necesidad de sentirse único Incen- } \\
\text { tivos económicos y premios } \\
\text { Buscar consejo Persuadir a otros } \\
\text { Regular emociones }\end{array}$ & $\begin{array}{l}\text { Mostrar identidad deseada Vincu- } \\
\text { lación social }\end{array}$ \\
\hline Beneficios para otros & $\begin{array}{l}\text { Altruismo } \\
\text { Agradecer a la empresa }\end{array}$ & Agradecer a la empresa \\
\hline
\end{tabular}

Fuente: elaboración propia.

\section{Motivaciones para eWOM de RSC}

A partir de la clasificación anterior, es necesario considerar varios aspectos para identificar las motivaciones que pueden estar presentes cuando los individuos deciden compartir la información de RSC generada por la empresa (WOM generado). En primer lugar, en este trabajo nos centramos en aquellas situaciones en las que la información de RSC es emitida por la empresa (WOM generado) como contenido de una acción de comunicación. Y, en segundo lugar, el individuo decide si se implica en su retransmisión (WOM transmitido) para satisfacer sus necesidades personales o para beneficiar a otros. Por tanto, la mejora del autoconcepto, mostrar una identidad deseada, la vinculación social y mostrar agradecimiento a la empresa pueden considerarse como las motivaciones que están presentes cuando los individuos deciden compartir dicha información de RSC.

\section{La mejora del autoconcepto}

Mejorar el autoconcepto se refiere a la tendencia a mantener una visión positiva de nosotros mismos (Dufner et al., 2018). Las personas llevan a cabo acciones que conducen a una autoevaluación positiva. A través de dichas acciones, el individuo puede recibir una retroalimentación positiva que le permita reafirmarse en atributos 
positivos como inteligencia, habilidad, astucia u honestidad (Ho y Dempsey, 2010). En este sentido y de acuerdo con Berger (2014), los individuos hablan con otros sobre sus experiencias de consumo positivas motivados por el reconocimiento y la recompensa que les permite reafirmar su autoconcepto de "buenos compradores". En este sentido, compartir información sobre RSC puede ayudar al consumidor a mejorar su autoconcepto por contribuir a la difusión de las acciones de RSC y asociar su nombre a acciones benévolas y de ayudar a la sociedad.

\section{Mostrar una identidad deseada}

Mostrar una identidad deseada se refiere a la necesidad de las personas de ser percibidas de forma diferente (Berger, 2014; Berger y Heath, 2007). En este sentido, los productos que compran y consumen constituyen un elemento diferenciador por el valor simbólico que poseen (Grewal, Stephen y Coleman, 2018). Por ejemplo, consumidores que quieren ser percibidos como de clase social alta podrían evitar comprar determinadas marcas de productos que son populares en una baja clase social (Chung y Darke, 2006).

Los consumidores pueden transmiten información sobre dichos productos para revelar aspectos sobre su identidad (Chung y Darke, 2006). En otras palabras, la información que se transmite sobre experiencias de consumo permite a otros individuos hacer inferencias sobre determinados aspectos de la identidad del emisor (Berger, 2014). Por ejemplo, un individuo que comparte información específica y detallada sobre telefonía móvil puede desear mostrarse ante otros como un experto en tecnología (Berger, 2014).

De acuerdo con Grewal, Stephen y Coleman (2018), las redes sociales proporcionan una plataforma donde los individuos tienen una audiencia a la que pueden enviar señales para mostrar su identidad deseada. Por tanto, compartir información de RSC puede ayudar a los individuos a mostrar una identidad diferenciadora porque, en general, otros consumidores perciben como positivas las acciones de RSC (Marin y Ruiz de Maya, 2013), y a aquellos que hablan de ellas se les asocia con individuos responsables socialmente.

\section{Vinculación social}

La vinculación social se define como la necesidad de pertenecer a un grupo (Schutz, 1966). A través de las redes sociales, los individuos buscan integrarse con quienes tienen temas en común y, como resultado, comparten información que los mantendrá socialmente conectados (Hennig-Thurau et al., 2004). Por tanto, compartir información de RSC les servirá para reforzar puntos de vista compartidos y delimitar la pertenencia a grupos con características comunes, bien sea porque comparten 
el interés por las actividades de RSC o porque se identifican con la empresa y las actividades de RSC han sido determinantes en esa identificación (Marin y Ruiz de Maya, 2013).

\section{Ayudar a la empresa}

Los individuos pueden compartir información positiva no solo para satisfacer sus deseos personales, sino también para ayudar a la empresa (Hennig-Thurau et al., 2004). Por ejemplo, los consumidores suelen agradecer por una experiencia de consumo positiva de consumo compartiendo mensajes positivos sobre la empresa (Sundaram et al., 1998). De esta forma, cuando el consumidor perciba que la actividad de RSC desarrollada por la empresa es positiva, podrá sentir la necesidad de compartir dicha información al tiempo que ayudará a la empresa a minimizar el escepticismo que se genera cuando es ella misma la que comunica directamente las acciones de RSC (Kim, 2019).

\section{Metodología}

A partir de los aspectos expuestos en la revisión de la literatura, en este apartado se detalla el estudio llevado a cabo mediante el método de investigación de análisis de contenido. De acuerdo con la metodología empleada por los autores Bigné y Royo-Vela (2013) y López y López (2019), el desarrollo de un análisis de contenido comprende tres fases. La primera fase es el diseño, la segunda es de análisis y la tercera de interpretación e informe final (ver figura 1). A continuación, se describe cada una de las fases desarrolladas.

\begin{tabular}{ccccc}
\hline $\begin{array}{c}\text { Selección de la } \\
\text { población y objeto } \\
\text { de estudio }\end{array}$ & $\begin{array}{c}\text { Selección de la } \\
\text { muestra }\end{array}$ & $\begin{array}{c}\text { Selección de la } \\
\text { unidad de análisis }\end{array}$ & $\begin{array}{c}\text { Selección de las } \\
\text { categorías de } \\
\text { contenido }\end{array}$ & $\begin{array}{c}\text { Selección del } \\
\text { sistema de } \\
\text { codificación }\end{array}$ \\
\hline Fase de análisis \\
\hline Realizar codificación & Analizar los datos & Conclusiones \\
\hline Fase de final & \\
& & Redactar informe \\
\hline Interpretar los datos & &
\end{tabular}

Figura 1. Fases para el desarrollo de un análisis de contenido Fuente: Bigné y Royo-Vela (2013). 


\section{Selección de la población y objeto de estudio}

Para iniciar este análisis de contenido, la selección de la población y el objeto de estudio ha sido el sector de la moda por dos motivos. En primer lugar, la mayor parte de los trabajos de RSC se realizan en el ámbito de industrias que generan controversia con respecto a sus efectos sobre la población o el medio ambiente como, por ejemplo, el alcohol, el tabaco o la industria minera o petrolera. La industria de la moda no está exenta de polémica en la actualidad al ser un sector con unos procesos productivos que generan un alto impacto ambiental por los escándalos de utilización de mano obra infantil y de personal mal remunerado, e incluso por la sostenibilidad a medio y largo plazo para el planeta de un determinado tipo de consumo de ropa. Por ejemplo, el grupo Inditex, propietario de varias marcas de moda en España, se ha visto involucrado en algunos escándalos en relación con dichos aspectos. En este contexto, las empresas de moda cada vez ponen más empeño en comunicar tanto los esfuerzos que realizan para minimizar los impactos negativos de sus procesos productivos, como el resto actividades socialmente responsables que también llevan a cabo (Elving et al., 2015). Siguiendo con el ejemplo de Inditex, este grupo comunica públicamente en su página web un informe o memoria sobre los impactos ambientales y sociales generados cada año. Además, cada vez presta más atención a la creación de productos sostenibles, lo que se pone de manifiesto en el lanzamiento de la línea Join Life para la marca Zara, principal enseña delgrupo. El segundo motivo está relacionado con el uso de las redes sociales como estrategia de comunicación por parte de estas empresas. La moda es un sector con una alta participación en redes sociales, ya que ha encontrado en ellas una forma de mantener una comunicación directa y dinámica con sus consumidores (Hollebeek, Glynn y Brodie, 2014).

Del ranking de empresas del sector comercio al por menor de prendas de vestir en establecimientos especializados en España en el año 2017, publicado por www.eleconomista.es, encontramos que el $100 \%$ de las diez primeras empresas cuentan con perfil de Facebook: Zara, HEM, Bershka, Mango, CEA, Desigual, PullEBear, Primark, Stradivarius y Massimo Dutti. En los tres primeros lugares por número de seguidores a nivel mundial están H\&M con treinta y seis mil millones, Zara con veintiséis mil millones y Mango con diez mil millones.

\section{Selección de la muestra}

Para seleccionar la muestra nos basamos en el conjunto de empresas decomercio al por menor de prendas de vestir con mayor facturación en España en el año 2017 (citadas en el apartado anterior). Para las diez primeras, analizamos si habían realizado 
publicaciones con contenido de RSC en su página de Facebook durante el primer trimestre del año 2018. Solo Zara, Mango, HEM y CEA cumplían este requisito. Por tanto, fueron las marcas que se incluyeron en el análisis.

Para cada marca se identificó la última publicación que cumplía con la característica de contenido de RSC y se comparó con el resto de las publicaciones (aquellas que no incluían contenido de RSC) y que la marca había hecho durante esa misma semana. En total, durante el periodo analizado se obtuvo una muestra de treinta y cinco publicaciones.

\section{Selección de la unidad de análisis}

De acuerdo con Krippendorff (1990), la unidad de análisis equivale a lo que se va a observar o lo que va a ser estudiado. Para este caso, la unidad de análisis son las publicaciones de Facebook de cada una de las marcas de moda seleccionadas (Zara, Mango, HEM y CEA). En la tabla 3 se muestra, en la primera columna, la marca seleccionada. En la segunda columna, se muestra la fecha de la última publicación que había realizado la marca y que contenía información de RSC. En la tercera columna se indica el periodo analizado, que corresponde a la semana correspondiente según la última publicación con contenido de RSC. La última columna muestra el número de publicaciones analizadas.

Tabla 3. Selección de la unidad de análisis

\begin{tabular}{llcc}
\hline Marca & Periodo de análisis & Fecha de publicación del mensaje de RSC & Unidad de análisis \\
\hline Zara & Del 19 al 25 de marzo 2018 & $24 / 03 / 2018$ & 13 \\
\hline Mango & Del 09 al 15 de abril 2018 & $14 / 04 / 2018$ & 11 \\
\hline HEM & Del 16 al 22 de abril 2018 & $18 / 04 / 2018$ & 7 \\
\hline CEA & Del 16 al 22 de abril 2018 & $22 / 04 / 2018$ & 4 \\
\hline & Total de publicaciones analizadas & & 35 \\
\hline
\end{tabular}

Fuente: elaboración propia.

\section{Selección de las categorías de contenido}

Las categorías del contenido a analizar se refieren a un grupo de palabras o de elementos de comunicación con un significado similar (Bigné y Royo-Vela, 2013). Siguiendo el trabajo de López y López (2019) se delimitan las siguientes categorías de contenido a medir: 
a. Tipo de contenido de la publicación. Las marcas publican información sobre RSC e información sobre productos.

b. Formato de la publicación. Se encontraron tres tipos de formato: imagen, texto o videos.

c. Reacciones a la publicación. Facebook le permite al usuario dar "me gusta", compartir la publicación o hacer un comentario. Estas reacciones son cuantificadas de la siguiente forma:

- Número de "me gusta" obtenidos. Facebook le permite al usuario asignar un "me gusta" a cada publicación que haga la marca. En cada publicación podemos ver el número total de "me gusta" que ha recibido.

- Número de veces que se comparte. Facebook le permite al usuario enviar la publicación específica a otro(s) contacto(s) a través de la opción "compartir". En cada publicación podemos ver el número total de veces que se ha compartido dicha publicación.

- Número de comentarios. Facebook le permite al usuario escribir un mensaje en cada publicación a través de la opción "comentar". En cada publicación se puede ver el número total de comentarios queha recibido dicha publicación.

\section{Selección del sistema de codificación}

Para concluir la primera fase de diseño, se debe seleccionar un sistema de codificación de las unidades de análisis en función de las categorías predefinidas (Bigné y Rolo-Vela, 2013). El esquema de codificación para este estudio fue desarrollado de forma deductiva. A partir de la literatura previa, dos codificadores ajenos al objetivo del trabajo (mayo 2018) identificaron los distintos contenidos que publicaban las marcas, los tipos de formato de las publicaciones y las posibles reacciones de los consumidores al ver la publicación (ver tabla 4). Estos codificadores eran estudiantes de último curso de grado en Marketing con un gran conocimiento en redes sociales y que habían sido instruidos para la realización de análisis de contenido, ya que estaban utilizando dicha metodología deinvestigación para la realización de su trabajo de fin de grado.

\section{Resultados}

Con el objetivo de contrastar los resultados obtenidos, se expresan los resultados por categorías. Primero, se presenta la categoría tipo de contenido de la publicación, luego la categoría formato de la publicación y, por último, la categoría de reacciones 
que incluirá en su descriptor el número de reacciones que la red social Facebook ha registrado.

Como se presenta en la tabla 5, para la categoría tipo de contenido de la publicación se mide el número de publicaciones que incluían o no contenido relacionado con la RSC.

Tabla 4. Sistema de codificación por categoría

\begin{tabular}{lll}
\hline \multicolumn{1}{c}{ Categoría } & \multicolumn{1}{c}{ Valores considerados } & \multicolumn{1}{c}{ Codificación } \\
\hline Tipo de contenido de la publicación & Contiene información de RSC & Sí $=1 /$ No $=0$ \\
\hline & Contiene imagen & Sí $=1 /$ No $=0$ \\
\cline { 2 - 3 } Formato de la publicación & Contiene texto & Sí $=1 /$ No $=0$ \\
\cline { 2 - 3 } & Contiene video & Sí $=1 /$ No $=0$ \\
\cline { 2 - 3 } & Ha sido compartido & Sí $=1 /$ No $=0$ \\
\hline Contiene comentarios recibidos & Sí $=1 /$ No $=0$ \\
\cline { 2 - 3 } & Número de "me gusta" & Sin código \\
\cline { 2 - 3 } & Número de veces compartido & Sin código \\
\cline { 2 - 3 } & Número de comentarios recibidos & Sin código \\
\hline
\end{tabular}

Fuente: elaboración propia.

Tabla 5. Resultados categoría tipo de contenido de la publicación

\begin{tabular}{lllllll}
\hline \multicolumn{1}{c}{ Categoría } & Zara & Mango & HEM & CEA & Total \\
\hline Contiene información de RSC & 2 & 7 & 1 & 1 & 11 \\
\hline No contiene información de RSC & 11 & 4 & 6 & 3 & 24 \\
\hline Total & 13 & 11 & 7 & 4 & 35 \\
\hline
\end{tabular}

Fuente: elaboración propia.

En la tabla 6 se muestra la categoría formato de la publicación. Esta categoría mide los tres tipos de formato utilizados en la red social Facebook: imagen, texto y video.

Tabla 6. Resultados categoría tipo de contenido de la publicación 


\begin{tabular}{lcccccc}
\hline & \multicolumn{2}{c}{ Contiene información de RSC } & \multicolumn{3}{c}{ No contiene información de RSC } \\
\hline Marca & \multicolumn{3}{c}{ Formato de la publicación } & \multicolumn{3}{c}{ Formato de la publicación } \\
\hline Imagen & Texto & Video & Imagen & Texto & Video \\
\hline Zara & 2 & 0 & 0 & 7 & 0 & 4 \\
\hline Mango & 5 & 0 & 2 & 3 & 1 & 0 \\
\hline HEM & 1 & 0 & 0 & 6 & 0 & 0 \\
\hline CEA & 1 & 0 & 0 & 3 & 0 & 0 \\
\hline Total & 9 & 0 & 2 & 19 & 1 & 4 \\
\hline
\end{tabular}

Fuente: elaboración propia.

Para la categoría reacciones a la publicación se miden los tres tipos de reacciones que la red social Facebook permite realizar al usuario: "me gusta", compartir la publicación o hacer comentario. Teniendo en cuenta que Facebook cuantifica estas reacciones y el número es visible para todos los usuarios, se presentan los resultados para las publicaciones que contienen tanto información de RSC como para las publicaciones que no contienen información de RSC. Estos resultados se presentan en las tablas 7 y 8 .

Tabla 7. Resultados categoría reacciones a las publicaciones que contienen información de RSC

\begin{tabular}{ccccc}
\hline$N^{\circ}$ & Marca & $N^{\circ}$ de "me gusta" & $\begin{array}{c}N^{\circ} \text { de veces } \\
\text { compartido }\end{array}$ & $N^{\circ}$ de comentarios \\
\hline 1 & Zara & 227 & 16 & 11 \\
\hline 2 & Zara & 122 & 5 & 156 \\
\hline 3 & Mango & 582 & 13 & 4 \\
\hline 4 & Mango & 453 & 6 & 0 \\
\hline 5 & Mango & 138 & 14 & 9 \\
\hline 6 & Mango & 406 & 10 & 20 \\
\hline 7 & Mango & 342 & 7 & 2 \\
\hline 9 & Mango & 470 & 28 & 6 \\
\hline 10 & Mango & 127 & 16 & 233 \\
\hline 11 & HEM & 101 & 15 & 142 \\
\hline
\end{tabular}

Fuente: elaboración propia. 
Tabla 8. Resultados categoría reacciones a las publicaciones que no contienen información de RSC

\begin{tabular}{|c|c|c|c|c|}
\hline N. ${ }^{\circ}$ & Marca & N. de "me gusta" & $\begin{array}{l}\text { N. de veces } \\
\text { compartido }\end{array}$ & N. de comentarios \\
\hline 1 & Zara & 200 & 29 & 3 \\
\hline 2 & Zara & 111 & 36 & 3 \\
\hline 3 & Zara & 134 & 7 & 2 \\
\hline 4 & Zara & 89 & 4 & 1 \\
\hline 5 & Zara & 69 & 2 & 4 \\
\hline 6 & Zara & 55 & 4 & 1 \\
\hline 7 & Zara & 124 & 8 & 8 \\
\hline 8 & Zara & 98 & 6 & 1 \\
\hline 9 & Zara & 246 & 8 & 7 \\
\hline 10 & Zara & 78 & 4 & 2 \\
\hline 11 & Zara & 67 & 2 & 3 \\
\hline 12 & Mango & 479 & 35 & 9 \\
\hline 13 & Mango & 289 & 21 & 9 \\
\hline 14 & Mango & 1.000 & 29 & 10 \\
\hline 15 & Mango & 267 & 2 & 11 \\
\hline 16 & HEM & 399 & 12 & 15 \\
\hline 17 & HEM & 303 & 9 & 8 \\
\hline 18 & HEM & 408 & 15 & 4 \\
\hline 19 & HEM & 69 & 8 & 5 \\
\hline 20 & HEM & 260 & 12 & 4 \\
\hline 21 & HEM & 133 & 30 & 16 \\
\hline 22 & CEA & 72 & 2 & 0 \\
\hline 23 & CEA & 87 & 4 & 1 \\
\hline \multirow[t]{2}{*}{24} & CEA & 64 & 4 & 0 \\
\hline & Total & 5.101 & 293 & 127 \\
\hline
\end{tabular}

Fuente: elaboración propia. 
Para finalizar el análisis, en la tabla 9 se presenta el resumen de los datos obtenidos en la aplicación del método de investigación de análisis de contenido. Los resultados del análisis de contenido muestran que las publicaciones de las marcas de moda en Facebook, que incluían información relacionada con las actividades de RSC, obtuvieron más reacciones en la categoría "me gusta" $(280,27)$ que las que no contienen información de RSC $(212,54)$. También se aprecia que el número de comentarios obtenidos por las publicaciones de RSC $(21,18)$ superan ampliamente el número de comentarios recibidos por las otras publicaciones $(5,29)$. Sin embargo, no se aprecian diferencias entre los dos tipos de publicaciones en cuanto al número de veces que los consumidores han decidido compartir la publicación.

Tabla 9. Resumen de resultados del método análisis de contenido

\begin{tabular}{|c|c|c|c|c|c|c|c|}
\hline \multirow[t]{2}{*}{ Categoría } & \multicolumn{4}{|c|}{ Muestra según formato } & \multirow{2}{*}{$\begin{array}{l}\text { N. de } \\
\text { "me gusta" } \\
\text { Media }\end{array}$} & \multirow{2}{*}{$\begin{array}{c}N .^{\circ} \text { de veces } \\
\text { compartido } \\
\text { Media }\end{array}$} & \multirow{2}{*}{$\begin{array}{c}N^{\circ} \text { de comentarios } \\
\text { recibidos }\end{array}$} \\
\hline & Imagen & Texto & Video & Total & & & \\
\hline $\begin{array}{l}\text { Contiene información } \\
\text { de RSC }\end{array}$ & 9 & 0 & 2 & 11 & 280,27 & 12,91 & 21,18 \\
\hline $\begin{array}{l}\text { No contiene informa- } \\
\text { ción de RSC }\end{array}$ & 19 & 1 & 4 & 24 & 212,54 & 12,21 & 5,29 \\
\hline
\end{tabular}

Fuente: elaboración propia.

\section{Discusión y conclusiones}

Consecuente con lo planteado en la revisión de la literatura, las publicaciones con contenido de información de RSC generan un mayor número de interacciones con los usuarios. El eWOM es una herramienta que no solo facilita la difusión de información corporativa relacionada con productos y servicios, sino que también incrementa la transmisión de mensajes de RSC de la empresa. Además, con el análisis de contenido realizado se muestran indicios de que la información de RSC parece despertar un mayor interés en los consumidores en términos de interacciones con la publicación como "me gusta", número de veces que se comparte y número de comentarios que se reciben en comparación con los contenidos publicados con otro tipo de información comercial.

Internet y las redes sociales han facilitado la aparición de nuevas formas de generar comunicaciones corporativas para las empresas. Estas implementan diferentes estrategias de comunicación de contenidos en redes sociales como mecanismo para mantener una relación cercana con sus consumidores y lograr una amplia difusión de la información comercial y no comercial. Como resultado, las empresas 
esperan que los consumidores tengan reacciones más positivas ante los contenidos publicados y, por ende, ayuden a la empresa en la difusión.

En este sentido, las motivaciones que tienen los consumidores para compartir información tienen importantes implicaciones para comprender cómo la exposición a información sobre las actividades de RSC aumenta la probabilidad de que los consumidores generen reacciones más positivas ante dicha información, loquegenera una mayor interacción con las publicaciones. De esta forma, este trabajo contribuye a la literatura de comportamiento del consumidor al proponer que existe una mayor probabilidad de que los consumidores generen más interacciones en redes sociales cuando se encuentran con información de RSC frente a otro tipo de información. Los consumidores comparten la información de RSC y apoyan la estrategia de difusión de mensajes comerciales de la empresa, motivados por mejorar el concepto que tienen de sí mismos, la necesidad de mostrar una identidad deseada, buscar vinculación social y por mostrar agradecimiento hacia la empresa.

Esta investigación ofrece un análisis interesante y útil a nivel gerencial y académico. Las empresas se pueden beneficiar de una mayor disposición de sus consumidores para difundir información sobre sus acciones de RSC. Por ello, es importante para los directivos de marketing y comunicaciones corporativas conocer cuáles son las motivaciones que mueven a los individuos a difundir información no comercial de la empresa (como la RSC), de forma que puedan diseñar estrategias más eficientes para favorecer ese comportamiento de los consumidores.

\section{Limitaciones y líneas futuras}

La principal limitación de este trabajo es el carácter exploratorio del estudio. Se hace necesario la realización de otros estudios de tipo explicativo que permitan contrastar unas hipótesis de investigación con respecto a la intención de llevar a cabo eWOM sobre información de RSC. Otra limitación reside en el número reducido de empresas analizadas y en el hecho de que solo nos hemos centrado en el sector de la moda. Además, este trabajo se ha centrado en el eWOM transmitido, es decir, la intención de comunicar la información que ha sido generada por la empresa (por ejemplo, publicación en Facebook). Sin embargo, aunque es menos frecuente, los consumidores también pueden escribir un mensaje de RSC por propia iniciativa (eWOM generado), por lo que sería interesante analizar en qué media la información de RSC es susceptible de ser comunicada por parte de los consumidores.

Por tanto, para la literatura científica se abren líneas futuras de investigación asociadas a la importancia del rol de las redes sociales en la comunicación de 
RSC y los efectos que tiene para las empresas involucrar a los consumidores en la comunicación de sus iniciativas de RSC. Futuros trabajos deben abordar el rol de las motivaciones para compartir información de RSC, es decir, estudiar cuáles de las motivaciones identificadas en este trabajo están influyendo en que el individuo comparta información de RSC. Un estudio empírico con consumidores nos ayudaría a aportar más conocimiento en este sentido. En segundo lugar, es necesario estudiar si existen diferencia en cuanto a la intención de compartir información de RSC cuando se trata de un eWOM generado por la empresa o por el consumidor, ya que este trabajo solo se ha centrado en una de las perspectivas. Además, sería interesante considerar qué sucede en otras redes sociales como Instagram o Twitter, las cuales pueden tener otras connotaciones. En este sentido, sería interesante analizar si la información de RSC sería mejor percibida en unas redes o en otras. Por ejemplo, Instagram es percibida como una red más amigable que Twitter (Pittman y Reich, 2016). Por último, también sería de interés analizar qué tipo de interacción (por ejemplo, reaccionar, comentar, compartir) favorece más a la empresa en términos de difusión de sus contenidos de RSC.

\section{Referencias}

Asociación Española de Contabilidad y Administración de Empresas, AECA (2004). Marco conceptual de la responsabilidad social corporativa. Serie Responsabilidad Social Corporativa, Documento, (1).

Alhidari, A., lyer, P. y Paswan, A. (2015). Personal level antecedents of eWOM and purchase intention, on social networking sites. Journal of Customer Behaviour, 14(2), 107-125. https:// doi.org/10.1362/147539215X14373846805707

Appelman, A. y Sundar, S. S. (2016). Measuring message credibility: Construction and validation of an exclusive scale. Spaniy, 93(1), 59-79. https://doi.org/10.1177/1077699015606057

Comisión Europea (2011). A renewed EU strategy 2011-2014 for Corporate Social Responsibility. Brussels.

Berger, J. (2014). Word of mouth and interpersonal communication: A review and directions for future research. Journal of Consumer Psychology, 24(4), 586-607. https://doi.org/10.1016/j. jcps.2014.05.002

Berger, J., y Heath, C. (2007). Where Consumers Diverge from Others: Identity Signaling and Product Domains. Journal of Consumer Research, 34(2),121-134.

Bhandari, M. y Rodgers, S. (2018). What does the brand say? Effects of brand feedback to negative eWOM on brand trust and purchase intentions. International Journal of Advertising, 37(1), 125-141. https://doi.org/10.1080/02650487.2017.1349030

Bigné, E. y Royo-Vela, M. (2013). Metodología del análisis de contenido. En F. J. Sarabia (ed.), Métodos de investigación social y de empresa (pp. 503-521). Ediciones Pirámide. 
Cantallops, A. S. y Salvi, F. (2014). New consumer behavior: A review of research on eWOM and hotels. International Journal of Hospitality Management, 36, 41-51. https://doi.org/10.1016/j. ijhm.2013.08.007

Cha, M. K., Yi, Y. y Bagozzi, R. P. (2016). Effects of customer participation in corporate social responsibility (CSR) programs on the CSR-brand fit and brand loyalty. Cornell Hospitality Quarterly, 57(3), 235-249. https://www.researchgate.net/publication/288683286

Chatterjee, P. (2001). Online reviews: do consumers use them? Advances in Consumer Research, 28, 129-133. http://acrwebsite.org/volumes/8455/volumes/v28/NA-28

Chu, S. C., Lien, C. H. y Cao, Y. (2018). Electronic word-of-mouth (eWOM) on WeChat: examining the influence of sense of belonging, need for self-enhancement, and consumer engagement on Chinese travellers' eWOM. International Journal of Advertising, 1-24.

Chung, C. y Darke, P. (2006). The consumer as advocate: Self-relevance, culture, and word of- mouth. Marketing Letters, 17(4), 269-279. https://link.springer.com/article/10.1007/ s11002-006-8426-7

Duan, W., Gu, B. y Whinston, A. B. (2008). Do online reviews matter? An empirical investigation of panel data. Decision support systems, 45(4), 1007-1016. https://doi.org/10.1016/j.dss.2008.04.001

Dufner, M., Gebauer, J. E., Sedikides, C. y Denissen, J. J. (2018). Self-enhancement and psychological adjustment: A meta-analytic review. Personality and Social Psychology Review. https:// doi.org/10.1177/1088868318756467

Elving, W. J., Golob, U., Podnar, K., Ellerup-Nielsen, A. y Thomson, C. (2015). The bad, the ugly and the good: new challenges for CSR communication. Corporate Communications: An International Journal, 20(2), 118-127. https://doi.org/10.1108/CCIJ-02-2015-0006

Erkan, I. y Evans, C. (2016). The influence of eWOM in social media on consumers' purchase intentions: An extended approach to information adoption. Computers in Human Behavior, 61, 47-55. https://www.researchgate.net/publication/297729360

Grewal, L., Stephen, A. T. y Coleman, N. V. (2018). When Posting About Products on SocialMedia Backfires: The Negative Effects of Consumer Identity Signaling on Product Interest. Journal of Marketing Research, 56(2), 197-210. https://doi.org/10.1177/0022243718821960

Gvili, Y. y Levy, S. (2016). Antecedents of attitudes toward eWOM communication: differences across channels. Internet Research, 26(5), 1030-1051. 10.1108/intr-08-2014-0201

Hennig-Thurau, T., Gwinner, K., Walsh, G. y Gremler, D. (2004). Electronic word of mouth via consumer opinion platforms: What motivates consumers to articulate themselves on the Internet? Journal of Interactive Marketing, 18(1), 38-52. https://doi.org/10.1002/dir.10073

Ho, J. Y. y Dempsey, M. (2010). Viral marketing: Motivations to forward online content. Journal of Business research, 63(9-10), 1000-1006. https://doi.org/10.1016/j.jbusres.2008.08.010

Hollebeek, L. D., Glynn, M. S., y Brodie, R. J. (2014). Consumer brand engagement in social media: Conceptualization, scale development and validation. Journal of interactive marketing, 28(2), 149-165. https://doi.org/10.1016/j.intmar.2013.12.002 
Joa, C. Y., Kim, K. y Ha, L. (2018). What Makes People Watch Online In-Stream Video Advertisements? Journal of Interactive Advertising, 18(1), 1-14. https://doi.org/10.1080/15252019.2018.1 437853

Kam, L., Robledo-Dioses, K. y Atarama-Rojas, T. (2019). Fashion Films as a Particular Content of Fashion Marketing: An Analysis of its Nature in the Context of Hybrid Messages. Anagramas. Rumbos y sentidos de la comunicación, 17(34), 203-224. http://dx.doi.org/10.22395/angr.v17n34a10

Kim, S. (2019). The process model of corporate social responsibility (CSR) communication: CSR communication and its relationship with consumers' CSR knowledge, trust, and corporate reputation perception. Journal of Business Ethics, 154(4), 1143-1159. https://link.springer.com/ article/10.1007/s10551-017-3433-6

King, R., Racherla, P., y Bush, V. (2014). What We Know and Don't Know About Online Word-ofMouth: A Review and Synthesis of the Literature. Journal of interactive marketing, 28(3), 167-183.

Krippendorff, K. (1990). Metodología de análisis de contenido: Teoría y práctica. Paidós Comunicación.

Kwon, S., Ha, S. y Kowal, C. (2017). How online self-customization creates identification: Antecedents and consequences of consumer-customized product identification and the role of product involvement. Computers in Human Behavior, 75, 1 13. https://doi.org/10.1016/j. chb.2017.04.051

Lim, M. (2020). The negative impact of market-discrimination CSR: How do consumers respond to perceived company greed? Journal of General Management, 45(2), 93-106. https://doi. org/10.1177/0306307019875896

López, A. M., y López, M. (2019). Estudio omnicanal de las empresas minoristas del sector cosmético en España. Redmarka. Revista de Marketing Aplicado, 23(2), 19-41. https://www. researchgate.net/publication/338212376

López, L. M. (2018). Variables de la actividad de una marca en Twitter que influyen en el comportamiento de difusión de contenido de marca en su audiencia. Esic market, (161), 455 - 499. https://www.researchgate.net/publication/329337887

López, M. ySicilia, M. (2013). Boca a boca tradicional vs. electrónico. La participación como factor explicativo de la influencia del boca a boca electrónico. Revista Española de Investigación en Marketing ESIC, 17(1), 7-38. https://www.researchgate.net/publication/257138737

Marin, L. y Ruiz de Maya, S. (2013). The role of affiliation, attractiveness and personal connection in consumer-company identification. European Journal of Marketing, 47(3/4), 655 673. https:// www.researchgate.net/publication/263146908

Matus, P. (2018). Seeking stakeholders: responsibility and effectiveness as values in letters from CEOS in the CSR reports in Chile. Anagramas-Rumbos y sentidos de la comunicación, 17(33), 29-49. http://dx.doi.org/10.22395/angr.v17n33a2

Moon, S. J., Costello, J. P. y Koo, D. M. (2017). The impact of consumer confusion from eco-labels on negative WOM, distrust, and dissatisfaction. International Journal of Advertising, 36(2), 246 271. https://doi.org/10.1080/02650487.2016.1158223 
Nielsen. (2015). Global trust in advertising: Winning strategies for an evolving media landscape. https://www.nielsen.com/ssa/en/insights/report/2015/global-trust-in-advertising-2015/

Oeldorf-Hirsch, A. y Sundar, S. S. (2015). Posting, commenting, and tagging: Effects of sharing news stories on Facebook. Computers in Human Behavior, 44, 240-249. https://www.researchgate.net/publication/269728086_Posting_commenting_and_tagging_Effects_of_sharing_news_stories_on_Facebook

Pittman, M. y Reich, B. (2016). Social media and loneliness: Why an Instagram picture may be worth more than a thousand Twitter words. Computers in Human Behavior, 62, 155-167. https://doi. org/10.1016/j.chb.2016.03.084

Podium (2017). Annual Report: State of Online Reviews. Recuperado de https://go.podium.com/ podium-state-of-online-reviews/

Schutz, W. (1966). Firo: A three dimensional theory of interpersonal behavior. Consulting Psychologist Press, Inc.

Sen, S. y Lerman, D. (2007). Why are you telling me this? An examination into negative consumer reviews on the web. Journal of interactive marketing, 21(4), 76-94. https://doi.org/10.1002/ dir. 20090

Song, B. y Wen, J. (2019). Online corporate social responsibility communication strategies and stakeholder engagements: A comparison of controversial versus noncontroversial industries. Corporate Social Responsibility and Environmental Management. https://doi.org/10.1002/ csr. 1852

Stryja, C. y Satzger, G. (2018). Try Before You Buy: How to Design Information Systems to Enhance

Consumer Willingness to Test Sustainable Innovations. Journal of Technology Management $\mathcal{E}$ Innovation, 13(1), 19-26. https://doi.org/10.4067/S0718-27242018000100019

Suárez-Perales, I., Rivera-Torres, P. y Garces-Ayerbe, C. (2018). Consequences of innovative proactivity in environmental management 1. Universia Business Review, (57), 56-91. https:// zaguan.unizar.es/record/70714?ln=es

Sundaram, D. S., Mitra, K. y Webster, C. (1998). Word-of-Mouth Communication: A Motivational Analysis. In J. W. Alba \& J. W. Hutchinson (eds.), Advances in Consumer Research, Vol. 25. (pp. 527-531). Association for Consumer Research.

Trusov, M., Bucklin, R. E. y Pauwels, K. (2009). Effects of word-of-mouth versus traditional marketing: findings from an internet social networking site. Journal of marketing, 73(5), 90- 102. https://doi.org/10.1509/jmkg.73.5.90

Veloutsou, C., Dessart, L., y Morgan, A. (2015). Consumer engagement in online brand communities: a social media perspective. Journal of product $\mathcal{E}$ Brand Management, 21(1), 28-42.

Zhu, X., Kim, Y. y Park, H. (2020). Do messages spread widely also diffuse fast? Examining the effects of message characteristics on information diffusion. Computers in Human Behavior, 103, 37-47. https://doi.org/10.1016/j.chb.2019.09.006 